\title{
Factors Affecting the Use of Personal Protective Equipment's (PPE) Among Sterile Team Members During Surgical Procedures at St, Paul's Hospital Millennium Medical College and AaBET Hospital Operating Theatre, Addis Ababa / Ethiopia
}

\author{
Teshale Biku $^{1^{*}} \quad$ Altayewerk Mekonnen ${ }^{2 *} \quad$ Tangute Demas $^{2^{*}}$ \\ 1.Operating theatre Nursing Education Department St, Paul's hospital Millennium Medical College Addis Ababa \\ / Ethiopia \\ 2.Surgical Nursing Education Department St, Paul's Hospital Millennium Medical College Addis Ababa / \\ Ethiopia
}

\begin{abstract}
Background: Personal protective equipment's (PPE) in the health care are designed to protect health care workers from serious workplace injuries or illnesses. PPE provides a physical barrier between microorganism and wearer. It offers protection by preventing microorganism from contaminating hands, eyes, clothing, hair and shoes and also being transmitted to other patients and staff. Worldwide, three million health care workers (HCWs) experience per-cutaneous exposure to blood-borne viruses such as hepatitis B, hepatitis C and HIV per year. The use of PPE is essential in infection control and protects HCWs from acquiring dangerous infection and diseases of epidemic proportion.Objective: The objective of this study was to assess factors affecting the use of personal protective equipment's (PPE) among sterile team members during surgical procedures at St. Paul's Hospital Millennium Medical College, and AaBET, Hospital Operating theatre in Addis Ababa, Ethiopia, 2019.

Result: In all study area (SPHMMC, AaBET,) there are sufficient supply of PPE, but there are some factors that affect use of PPE. According to our respondents 52(36.36\%) were influence by hospital policy, $84(58.74 \%)$, were influenced by senior example, because seniors who are not using PPE properly, and $75(52.44 \%)$ were influenced by no time available to wear PPE during emergency cases. Recommendations: Each operation theater should have a specific complete written policy or standard operating procedures on proper attire that is known to all persons, continued education on use of PPEs and attachment of punishment to non-compliance. Also staffs need to update their experiences, and seniors should have responsibility for their act. In general, all health-care professionals and institutions have obligations to provide safe and quality health care and to avoid unintentional harm to patients.

Keywords:Personal protective equipment (PPE), St. Paul's Hospital Millennium Medical College, Operation room (OR),Addis Ababa burn emergency and trauma hospital(AaBET), Health care workers (HCWs), Universal precautions (Ups)

DOI: $10.7176 / \mathrm{JHMN} / 88-03$
\end{abstract}

Publication date: April $30^{\text {th }} 2021$

\section{Introduction}

The operating theatre is a dynamic and complicated area, where the safety of patients' undergoing surgery requires great priority. Health care professionals are spending their times and lives at work place and exposing their health to different type of infections. Thus infections in working surrounding can challenge the workers' capability to care for their health, and well-being. (1) Personal protective equipment (PPE) in the health care are designed to protect health care workers from serious workplace injuries or illnesses (2). PPE provides a physical barrier between microorganism and wearer. It offers protection by preventing microorganism from contaminating hands, eyes, clothing, hair and shoes and also being transmitted to other patients and staff $(3,4)$.

Lack of comfort/fit and fogging of eyewear and the need to scratch as well as young age and lack of safety training has been reported as the main factors to prevent the proper use of PPE. Appropriate use of PPE is the easiest way to prevent contact from secretions and transfer of pathogens. It's important to assess the level of compliance with use of PPE by the various HCWs who make direct contact with patients. (5)

PPE should be used by health care workers who provide direct care to patients and who work in situations where they may have contact with blood, body fluids, excretions or secretions. Supporting staff including medical aides, cleaners, and laundry staff in situations where they may have contact with blood, body fluids, secretions and excretions should use PPE, and laboratory staff, who handle patient specimens; and family members who provide care to patients are in a situation where they may have contact with blood, body fluids, secretions and excretions should use PPE. If the work done by health care workers is not equipped with the necessary PPE in accordance with the established procedures, it will potentially transmit infectious diseases for both patients and health care workers (6).

In the developing countries, the HCWs are at greater risk due to sub-optimal infection control practices like 
lack of equipment, training, compliance with Ups (9) Limited data are available from low and middle income countries, but the prevalence of HAIs is estimated to be between $5.7 \%$ and $19.1 \%$. The increased burden of HAIs in LMICs affects especially high-risk populations, such as patients with surgical interventions and patients admitted to intensive care units (10). In a study conducted in India by Archana, et al., 2018 on appropriate use of PPE among the HCWs were only $18.1 \%$ and among the HCWs appropriate use of gloves, mask, apron, gown and hair cover was $100 \%$, but the use of goggles and shoe cover was very low. While doing procedures use of gloves was $100 \%$, mask was $95.6 \%$, but the use of other PPE was very less to the extent that the use of goggles $4.4 \%$, apron $62.3 \%$, gown $44.3 \%$, hair cover $57.9 \%$ and shoe cover $27.3 \%$ (5).

On studying factors affecting adherence to usage of PPE among participants by Hakim, S. et al. in Egypt in 2016 the percentage of nurses was significantly higher than that of physicians to believe the following factors have strong influence: previous inoculation, past experience, peer or senior example, wearing PPE interferes with doing their work tasks, availability of PPE at work, urgent patient needs. While physicians found that hospital policy, not enough time, and patient objection are factors with a significant strong influence on adherence to PPE usage. Although most of them agreed that usage of PPE can reduce the work hazards (45.7\%), the highest percentage of them stated that they only (sometimes) used PPE (41.2\%). Moreover, most of them disagreed that a hospital policy on PPE usage will increase adherence. (4)

This study was conducted in the operating rooms of St. Paul's Hospital Millennium Medical College (SPMMC), Addis Ababa burn, emergency and trauma hospital (AaBET), and Michu infertility clinic on staffs who are working in these units during surgical procedure. The research made for a study in Ethiopia on the issue indicated no available study in this area, hence this study was conducted assess factors that affect the use of PPE in operating theatre sterile team member staffs during surgical procedure.

\section{Methods \\ Study area}

The study was conducted in St. Paul's Hospital Millennium Medical College, which is managed by Ministry of Health. The college has more than 2, 800 clinical, academic and administrative and support staffs that provide medical specialty services to patients who are referred from all over the country, teaching medicine and nursing students and doing basic and applied researches. While the inpatient capacity is more than 700 beds, the College sees an average of 1, 200 emergency and outpatient clients daily.

\section{Study Period and design}

The study period was from the June2019 to July 2019 at St. Paul's Millennium Medical College, AaBET hospital and Michu infertility clinic. And the study design was, Institutional based cross-sectional study was conducted by using quantitative research method.

\section{Source population}

All health care workers who were participated for surgery as a sterile person at St. Paul's Hospital Millennium Medical College, AaBTE hospital, and Michu infertility clinic operation rooms.

\section{Study subjects}

The study subjects were randomly selected, operation room sterile team member staffs who were worked in St, Paul's Hospital Millennium Medical College, ABTE hospital, and Michu infertility clinic operation rooms from June2019 to July 2019

\section{Sample size determination}

The sample size was calculated by using single population proportion formula by considering the following assumptions: Since there is no known prevalence study in Ethiopia on PPE- $\mathrm{P}=50 \%$

Level of confidence $=95 \%$

Margin of error $(d)=5 \%$

$$
\begin{aligned}
& \mathrm{n}=\frac{(\mathrm{Z} \alpha / 2)^{2} \mathrm{P}(1-\mathrm{P})}{\mathrm{d}^{2}} \\
& \mathrm{n}=\underline{(1.96)^{2}} \frac{\mathrm{X} 0.5(1-0.5)}{(0.05)^{2}}=384
\end{aligned}
$$

Because the source population is less than 10,000 (207) by using reduction formula the calculated sample size will be: 


$$
\begin{aligned}
\mathrm{n} & =-\frac{\mathrm{n}}{1+\underline{\mathrm{n}}} \\
& =\frac{384}{1+\frac{384}{207}} \\
& =135
\end{aligned}
$$

By adding $10 \%$ non-response rates $=135+14$

The total sample size $=\mathbf{1 4 9}$

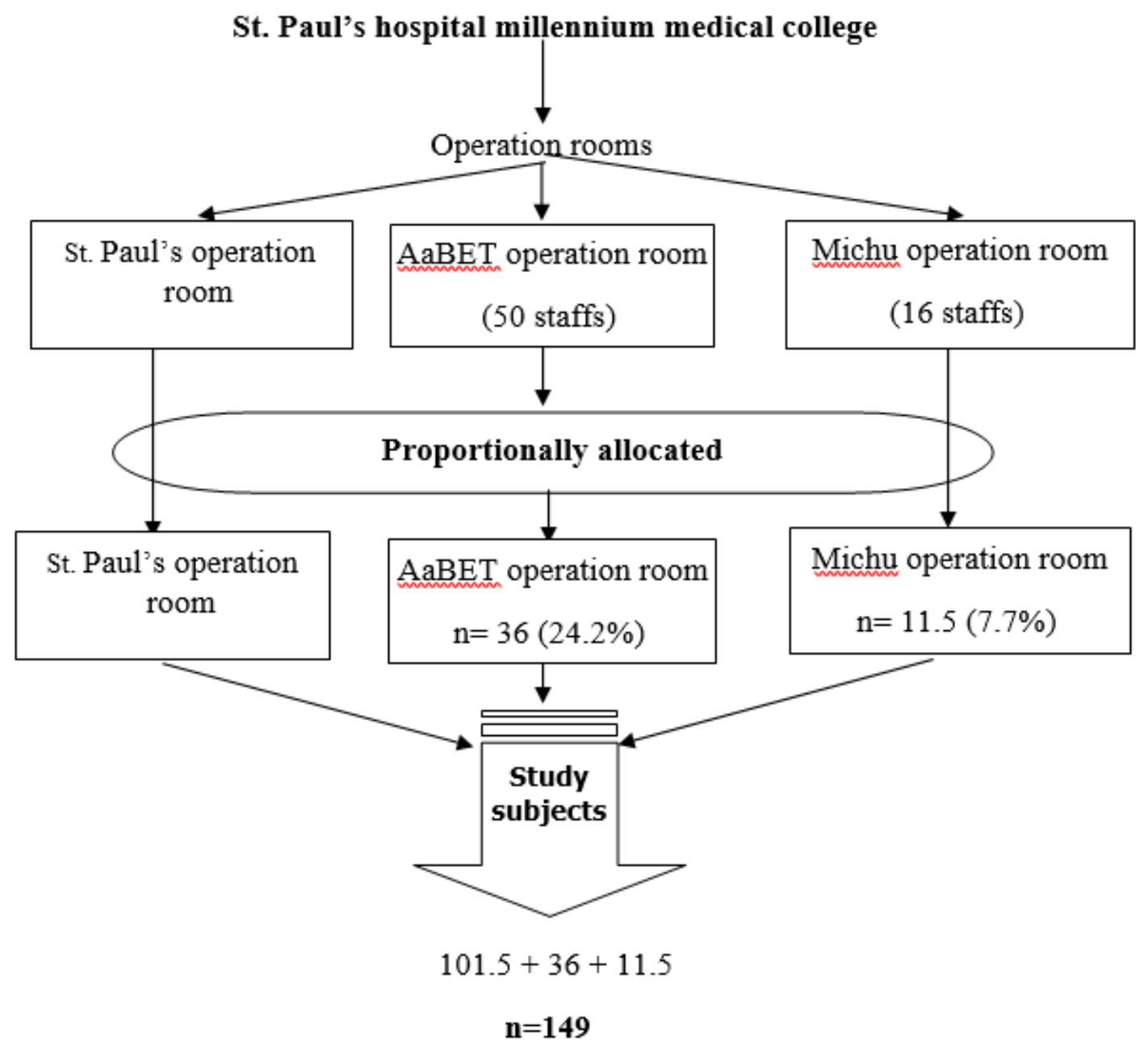

Figure 1: Schematic representation of sampling procedure for Assessment of Factors Affecting the use of Personal Protective Equipment's among operating theatre sterile team member staffs during surgical procedure in St. Paul's Hospital Millennium Medical College, AaBET hospital and Addis Ababa, Ethiopia, 2019.

\section{Tool for data collection}

The self-made questionnaire, which is developed by reviewing different literatures $(2,4-6,12,14,16,19)$, was used after making pretest in Tikur Anbessa Specialized Hospital to assess its clarity, instrument simplicity, applicability, flow and consistency of the data collection tools and to provide feedback about the questionnaire and standardize the data collection tool.

\section{Data collection technique}

After getting the study participants consent, the researchers distribute the questionnaire to be filled by the study participants 


\section{Operational definition}

- PPE:- Materials prepared to prevent health workers from different hazards

- HCWs :- Trained in health profession to work in health institutions

- Sterile team :- Surgical team members assigned to perform operation

- Factors :- Any causes which can affect the normal process

\section{Eligibility criteria}

Inclusion: - All staffs assigned and worked in the three operation rooms from the month June 2019 to July 2019

Exclusion: - . Those who were in sick leave or on annual vacation

. Staffs who were had less than 6 months of work experience in the operating theatre

\section{Pretest}

The data collection tool was pre-tested on 15 operation room staffs in Tikur Anbesa Specialized Hospital before the actual time of data collection to assess clarity, instrument simplicity, applicability, flow and consistency of the data collection tools and to provide feedback about the questionnaire and standardize the data collection approach.

\section{Data analysis}

Data analysis was made by coding each question to facilitate the entry of data into the result table, grouping the questions based on the variables studied, and questionnaire result into the table, processing the data into the form of frequency, percentage by using manual scientific calculator and display the results in the form of tables, diagrams, and drawings, and the last is to make the discussion and conclusion of the data obtained.

\section{Ethical Considerations}

In order to follow the ethical and legal standards of scientific investigation, this study was conducted after the submission and approval by the college research and ethics review committee and the Institutional Review Board (IRB) of the college to obtain ethical approval and clearance. Permission and supportive letter were requested from the provost of the institute before data collection. Participation was voluntary and information collected anonymously after obtaining signed consent from each respondent by assuring confidentiality throughout the data collection period. Participants notified of the objective of the study and their right to refuse to answer the questionnaires and given the right to stop or withdraw at any time of data collection.

\section{Result of the Study}

The questionnaire distributed to 149 persons according to our sample size but we got only 143 completed questionnaires, therefore the response rate was $95.97 \%$.

Among the respondents $89(62 \%)$ was categorized from age 25 to 35 , with the mean age of 30 . More than half $82(57 \%)$ was female, whereas $61(43 \%)$ was male. Regarding their work experience $102(71.32 \%)$ of the respondents were worked from 1 to 9 years. $83.19 \%$ of the respondents were nurses. Training given to $22 \%$ of the respondents. Most of the respondents working area were major operation rooms.

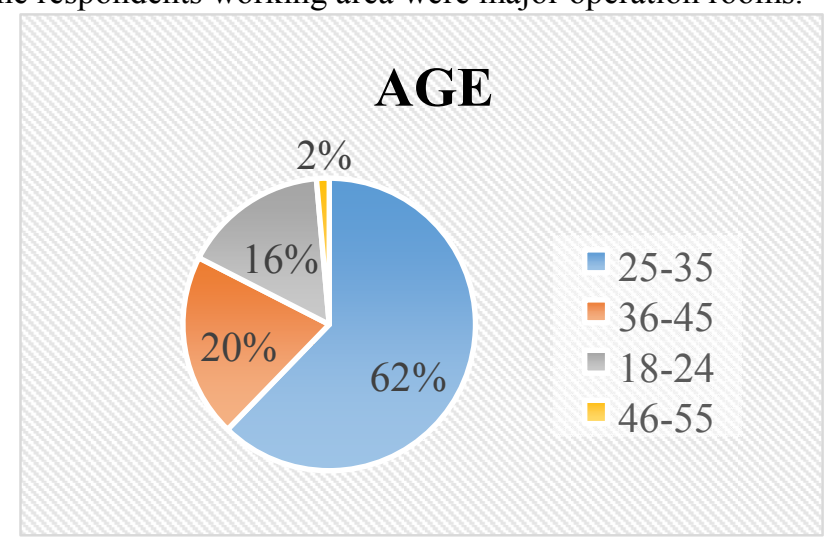

Figure 2: Age distribution of respondents for the use of Personal Protective Equipment's among operating theatre sterile team member staffs during surgical procedure in St. Paul's Hospital Millennium Medical College, and affiliated sites. Addis Ababa, Ethiopia, 2019. 


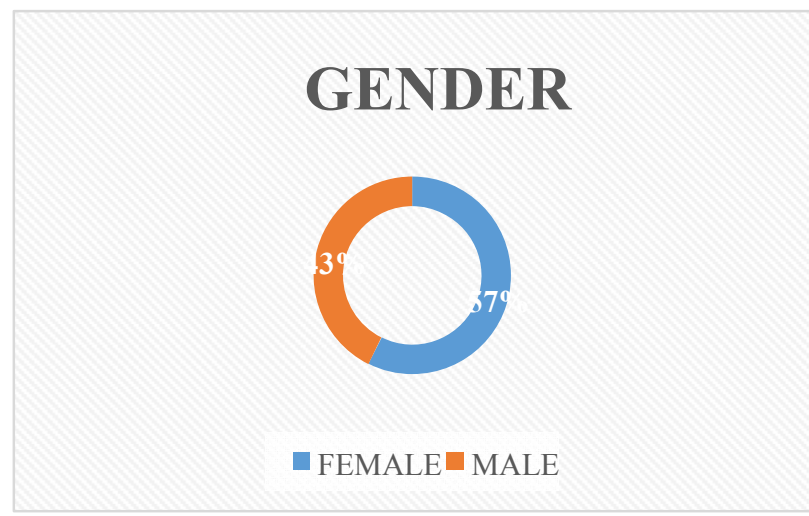

Figure 3: Gender distribution of respondents for the use of Personal Protective Equipment's among operating theatre sterile team member staffs in St. Paul's Hospital Millennium Medical college, and affiliated sites . Addis Ababa, Ethiopia, 2019.

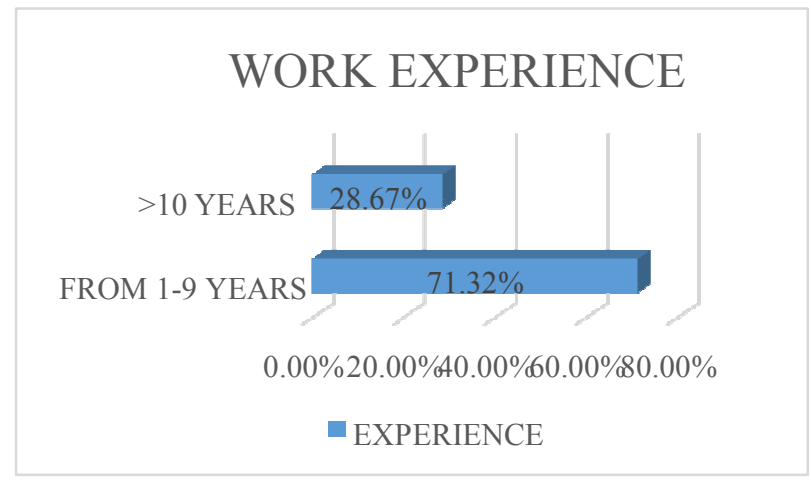

Figure 4: Work experience of respondents for the use of Personal Protective Equipment's among operating theatre team member staffs in St. Paul's Hospital Millennium Medical college, and affiliated sites, Addis Ababa, Ethiopia, 2019.

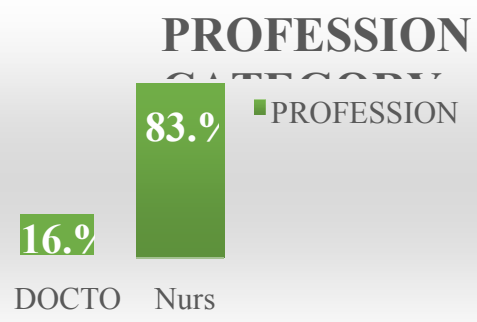

Figure 5: Profession category of respondents for the use of Personal Protective Equipment's among operating theatre sterile team staffs in St. Paul's Hospital Millennium Medical College, and affiliated sites. Addis Ababa, Ethiopia, 2019.

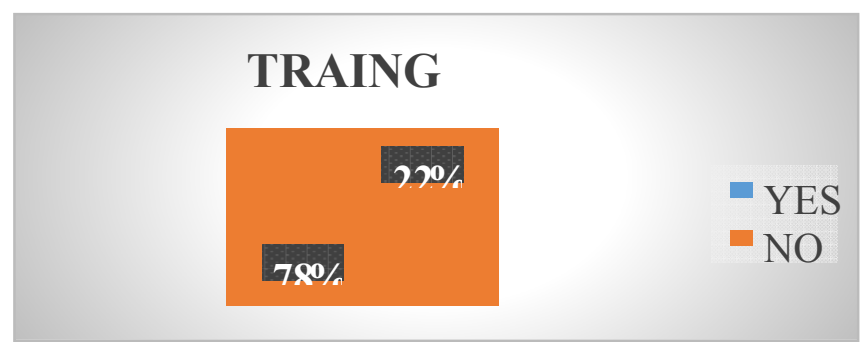

Figure 6: Respondents who took training for the use of Personal Protective Equipment's 22\% among operating theatre sterile team members, in St. Paul's Hospital Millennium Medical college, AaBET Hospital, and Michu infertility clinic. Addis Ababa, Ethiopia, 2019 


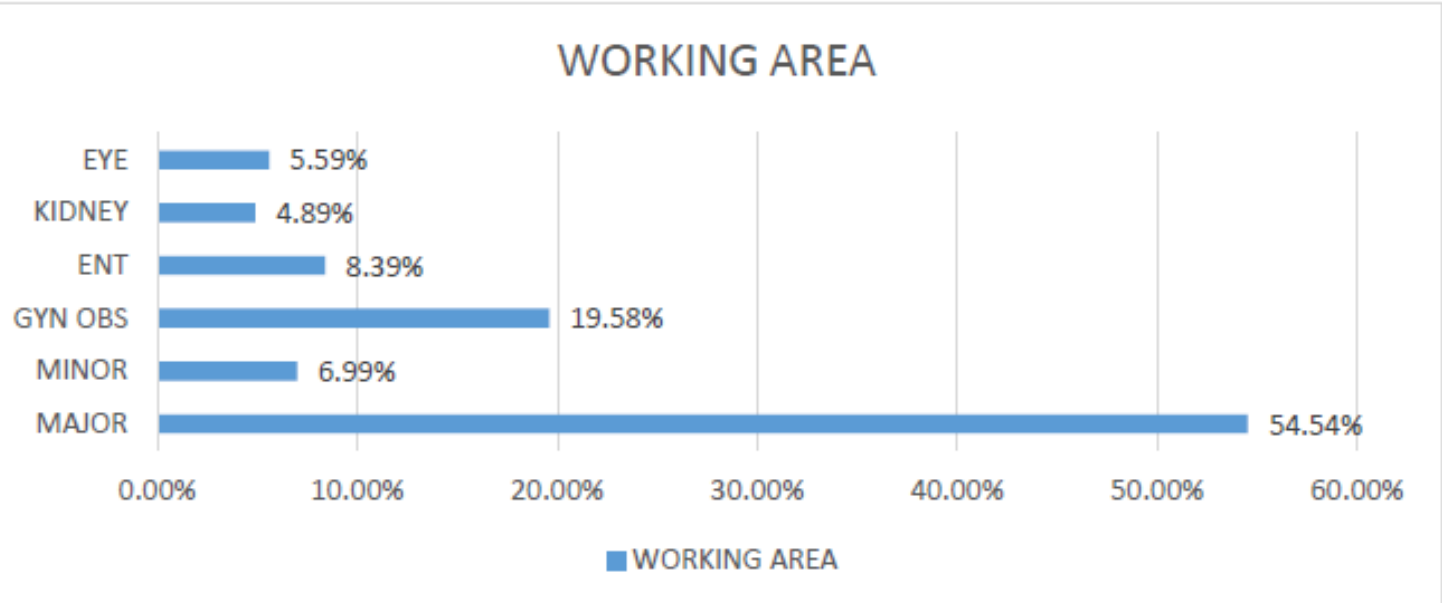

Figure 7: Working area of respondents in St. Paul's Hospital Millennium Medical College, AaBET hospital and Michu Infertility clinic operating rooms, Addis Ababa, Ethiopia, 2019.

Table 1: Availability of PPE in St. Paul's hospital and AaBET hospital Operating rooms during surgical procedures, Addis Ababa, Ethiopia, 2019.

\begin{tabular}{|c|c|c|c|c|c|c|c|}
\hline \multirow{2}{*}{ Hospitals } & \multicolumn{7}{|c|}{ Availability of PPE } \\
\hline & $\begin{array}{c}\text { Gloves } \\
\text { double }\end{array}$ & Mask & Eye google & Gown & $\begin{array}{c}\text { Boots/shoe } \\
\text { cover }\end{array}$ & Apron & Hair cover \\
\hline AaBET & Yes & Yes & Not enough & Yes & Yes & Not enough & Yes \\
\hline Michu & Yes & Yes & Not enough & Yes & Yes & Not enough & Yes \\
\hline St. Paul's & Yes & Yes & Not enough & Yes & Yes & Not enough & Yes \\
\hline
\end{tabular}

Eye goggle and apron available $65 \%$ but others PPE available sufficiently.

Table 2: Items practiced in OR during surgical procedure among operating theatre sterile team member staffs at St. Paul's Hospital Millennium Medical college, AaBET hospital and Michu infertility clinic in Addis Ababa, Ethiopia, 2019.

\begin{tabular}{|l|l|l|}
\hline Item & Types of PPE used & Percentage \\
\hline 2 pair of gloves & 108 & $75.52 \%$ \\
\hline Mask & 143 & $100 \%$ \\
\hline Eye goggle & 4 & $2.7 \%$ \\
\hline Gown & 143 & $100 \%$ \\
\hline Boots/shoe cover & 143 & $100 \%$ \\
\hline Hair cover & 143 & $100 \%$ \\
\hline Apron & 27 & $18.88 \%$ \\
\hline
\end{tabular}

Double gloves were worn by $75.52 \%$ of relevant sterile team members. Most of the respondents, more than $97 \%$ are not wearing eye protection and Apron was worn by only $18.88 \%$.

Table 3: Senior examples for the use of Personal Protective Equipment's among operating theatre staffs during surgical procedure at St. Paul's Hospital Millennium Medical college, AbET hospital and Michu clinic in Addis Ababa, Ethiopia, 2019.

\begin{tabular}{|l|l|l|}
\hline Its influence & Total number & Percentage \\
\hline Strong influence & 84 & $58.74 \%$ \\
\hline Some influence & 32 & $22.37 \%$ \\
\hline Limited influence & 11 & $7.69 \%$ \\
\hline No influence & 16 & $11.18 \%$ \\
\hline total & 143 & $100 \%$ \\
\hline
\end{tabular}

$58.74 \%$ of respondents strongly influenced by senior example.

Table 4: Hospital policy for personal Protective Equipment's, during surgical procedure in St. Paul's Hospital 
Millennium Medical College, and affiliated sites. Addis Ababa, Ethiopia, 2019.

\begin{tabular}{|l|l|l|}
\hline Its influence & Total number & Percentage \\
\hline Strong influence & 52 & $36.36 \%$ \\
\hline Some influence & 44 & $30.76 \%$ \\
\hline Limited influence & 34 & $23.77 \%$ \\
\hline No influence & 13 & $9.09 \%$ \\
\hline total & 143 & $100 \%$ \\
\hline
\end{tabular}

$36.36 \%$ respondent influenced by hospital policy.

Table 5: No time available to wear PPE among operating theatre sterile team member staffs during surgical procedure at St. Paul's Hospital Millennium Medical college, AbET hospital and Michu clinic in Addis Ababa, Ethiopia, 2019.

\begin{tabular}{|l|l|l|}
\hline Its influence & Total number & Percentage \\
\hline Strong influence & 61 & $42.65 \%$ \\
\hline Some influence & 33 & $23.07 \%$ \\
\hline Limited influence & 37 & $25.87 \%$ \\
\hline No influence & 22 & $15.38 \%$ \\
\hline total & 143 & $100 \%$ \\
\hline
\end{tabular}

$42.65 \%$ respondents influenced by no time available to wear PPE.

Table 6: Use of PPE in Emergency cases among operating theatre staffs during surgical procedure at St. Paul's Hospital Millennium Medical college, AaBET hospital and Michu clinic in Addis Ababa, Ethiopia, 2019.

\begin{tabular}{|l|l|l|}
\hline Its influence & Frequency & Percentage \\
\hline Strong influence & 75 & $52.44 \%$ \\
\hline Some influence & 46 & $32.16 \%$ \\
\hline Limited influence & 15 & $10.48 \%$ \\
\hline No influence & 7 & $4.89 \%$ \\
\hline total & 143 & $100 \%$ \\
\hline
\end{tabular}

Most of the respondents are highly influenced by emergency cases.

Availability of PPE is sufficient but they did not use properly. Respondents believe that the following factors have strong influence: hospital policy $36.36 \%$, senior example, $58.74 \%$, and no time available to wear PPE $42.65 \%$ and $52.44 \%$ in emergency cases.

\section{DISCUSSION}

Personal protective equipment (PPE) in the health care are designed to protect health care workers from serious workplace injuries or illnesses. PPE provides a physical barrier between micro-organism and wearer. It offers protection by preventing microorganism from contaminating hands, eyes, clothing, hair and shoes and also being transmitted to other patients and staff. Worldwide, three million health care workers (HCWs) experience percutaneous exposure to blood-borne viruses such as hepatitis B, hepatitis $\mathrm{C}$ and HIV per year.

In this research 78\% respondents have had no training. Another study conducted in Uganda by Okello, et al., in 2017 which shows about $30.5 \%$ of the hospital employees have had no training regarding PPE use,however,thisstudy has given better result compare with our study.

The result of our study showed $75.52 \%$ of the respondents wear 2 pairs of gloves, $100 \%$ wear mask, $2.7 \%$ eye protection, $100 \%$ wearing gown, $100 \%$ wearing boots/shoe cover, $100 \%$ wearing hair cover and $18.88 \%$ of wear apron the result less than a study conducted in India by Archana, et al., 2018 on appropriate use of PPE among the HCWs appropriate use of gloves, mask, apron, gown and hair cover was $100 \%$, but the use of goggles $4.4 \%$ and apron $62.3 \%$. (5).

The result of this study less than pervious study conducted in India, this is due to factors like hospital policy $36.36 \%$, senior example $58.74 \%$, and no time available to wear PPE $42.65 \%$ and use of PPE in emergency cases $52.44 \%$.

\section{Conclusion and Recommendation Conclusion}

The research revealed about Factors Affecting the use of Personal Protective Equipment's among Operating theatre sterile team member staffs in SPMMC. The study data were gathered using questionnaires which were filled by 143 staffs who are working at St. Paul's Hospital Millennium Medical College operation rooms, AaBET hospital, and Michu infertility clinic. The respondents have different characteristic like age, gender, work experience, profession category, qualification, taking training and working area. From the questionnaire we got that, most of the respondents were, categorized from age 25 to 35 
- Females were (57\%)

- They have experience from 1 to 9 years

- Most of them are nurse and midwife with BSC

- Half of the respondents took training

- Most of the respondents working area were in major operating rooms.

Double gloves were worn by $75.52 \%$ of relevant health care workers. Most of the respondents were not wearing eye protection $2.7 \%$ and Apron were worn by $18.88 \%$.

There were enough PPE in the study area, but there were some factors that affect the use of PPE as follows. Respondents

- Influenced by hospital policy $36.36 \%$,

- Senior example $58.74 \%$,

- No time available to wear PPE $42.65 \%$

- $\quad$ Use of PPE in emergency cases $52.44 \%$.

\section{ACKNOWLEDGEMENTS}

The authors would like to thank St, Paul's Hospital Millennium Medical College and AaBET Hospital for their willingness and support during the data collection process. We would like also to thank Mr Tekalign Zewdie for the English language editing of the manuscript.

\section{Author Contributions}

All authors contributed toward to the study design, data collection, data analysis, drafting and final approval of the version to be published, as well agree to be accountable for all aspects of the work.

\section{Disclosure}

The authors report no conflicts of interest in this work

\section{REFERENCES}

1. Union E. Priorities for occupational safety and health research in Europe: 2013-2020. 2013.

2. Aguwa EN, Arinze-Onyia SU, Ndu A. Use of Personal Protective Equipment among Health Workers in Tertiary Health Institution, South East Nigeria: Pre- Ebola Period. IJHSR. 2016; 6(8):12-8.

3. WHO. Practical Guidelines for Infection Control in Health Care Facilities. 2004.

4. Hakim S, Abouelezz N, El Okda E. Use of personal protective devices among health care workers in a teaching hospital in Cairo, Egypt. Egyptian J Occupational Med. 2016; 40(2):287-300.

5. Archana Lakshmi PA, Jennifer H G, Stanly A M, Paul CM. A study on personal protective equipment use among health care providers, Tamil Nadu. International Journal of Community Medicine and Public *Health. 2018; 5(5).

6. Daugherty EL, Perl TM, Needham DM, Rubinson L, Bilderback A, Rand CS. The use of personal protective equipment for control of influenza among critical care clinicians: A survey study. Crit Care Med. 2009; 37(4):1210-6.

7. WHO. The word health report: Reducing risks, promoting healthy life. 2002. 8. Currin E. Reducing the risk of health care acquired infection. Nursing standard. 2001; 16 (1):45-52.

8. Sagoe-Moses C, Pearson RD, Perry J. Risks to health care workers in developing countries. N Engl J Med. 2001; 345: 538-41.

9. WHO. Guidelines on Core Components of Infection Prevention and Control Programmes at the National and Acute Health Care Facility Level. 2016.

10. Sangwan BR, Kotwal A, Verma AK. Occupational Exposure to Blood and Body Fluids amongst Health Care Workers in a Teaching Hospital of the Armed Forces. Medical Journal Armed Forces India. 2011; 67(1):21-4.

11. Ayu CP. Evaluation of Knowledge and Compliance of Nurses on the Use Personal Protective Equipment (PPE) in Intensive Care Unit (ICU) RSUD Panembahan Senopati Bantul Yogyakarta. Jurnal Medico eticolegal and Manajemen Rumah Sakit. 2017; 6(3).

12. Kermode M, Jolley D, Langkham B, Thomas MS, Crofts N. Occupational exposure to blood and risk of bloodborne virus infection among health care workers in rural north Indian health care settings. Am J Infect Control. 2005; 33(1):34-41.

13. Okello TR, Kansime K, Odora J, Apio JA, Pecorella I. Barriers and factors affecting personal protective equipment usage in St. Mary's Hospital Lacor in Northern Uganda. East and Central African Journal of Surgery. 2017; 22(1).

14. Kotwal A, Taneja D. Health Care Workers and Universal Precautions:Perceptions and Determinants of 
Non-compliance. Indian J Community Med. $2010 ; 35$ (4):526-8.

15. Hu X, Zhang Z, Li N, Liu D, Zhang L, He W, et al. Self-reported use of personal protective equipment among Chinese critical care clinicians during 2009 H1N1 influenza pandemic. PLoS One. 2012; 7(9): e44723.

16. Aisien AO, Ujah IA. Risk of blood splashes to masks and goggles during cesarean section. Med Sci Monit. 2006; 12(2).

17. Deniz A, Kim LE, Parks RL, L'Ecuyer PB, Mutha S. Use of personal protective equipment surgery. Infection and operating room behaviors in four surgical subspecialties: personal protective equipment and behaviors in surgery. Infection Control and Hospital Epidemiology. 1999; 20:110-4.

18. Luo Y, He GP, Zhou JW, Luo Y. Factors impacting compliance with standard precautions in nursing, China. Int J Infect Dis. 2010; 14(12): e1106-14.

19. Implementation Manual -WHO Surgical Safety Checklist (First Edition). United States, World Health Organization, 2008. WHO/IER/PSP/2008.05. 